\title{
Disparidade urbano-rural na utilização de serviços de saúde em município de pequeno porte
}

\author{
Urban-rural disparity in health services utilization in a small municipality
}

\begin{abstract}
Como citar este artigo:
Vieira EWR, Dutra IR, Cerqueira LJ, Gazzinelli A. Urban-rural disparity in health services utilization in a small municipality. Rev Rene. 2020;21:e42458. DOI: https://doi.org/10.15253/2175-6783.20202142458
\end{abstract}

DEd Wilson Rodrigues Vieira ${ }^{1}$
(DIzabela Rocha Dutra ${ }^{2}$
(D) Lana Jaya Cerqueira $^{1}$
(D)Andréa Gazzinelli ${ }^{1}$

${ }^{1}$ Universidade Federal de Minas Gerais.

Belo Horizonte, MG, Brasil.

${ }^{2}$ Universidade Federal dos Vales do Jequitinhonha e Mucuri.

Diamantina, MG, Brasil.

\section{Autor correspondente:}

Ed Wilson Rodrigues Vieira

Avenida Professor Alfredo Balena 190,

Escola de Enfermagem, Sala 418 - Santa Efigênia,

CEP: 30.130-000. Belo Horizonte, MG, Brasil.

E-mail: edwilsonvieira@ufmg.br

\begin{abstract}
RESUMO
Objetivo: analisar a existência de disparidade urbano-rural na utilização de serviços de saúde por pessoas vivendo em um município de pequeno porte. Métodos: estudo epidemiológico transversal com amostra representativa e aleatória de pessoas com 18 anos ou mais de idade na área urbana $(n=1.235)$ e toda a população de um distrito rural $(n=190)$. Utilizou-se o questionário sobre utilização de serviços da Pesquisa Nacional por Amostra de Domićlios. A análise considerou modelos de regressão logística para examinar fatores associados à utilização nos 30 dias anteriores ao estudo. Resultados: a taxa de utilização pelas pessoas da área urbana foi significativamente maior que da área rural $(45,0 \%$ vs $15,8 \%$ ). Mulheres e aqueles que reportaram sintomas de doenças no período e hipertensão arterial tiveram mais chances de utilização. Conclusão: pessoas residentes em área rural utilizam menos serviços de saúde quando comparadas àquelas da área urbana quando estão sob a mesma organização municipal de saúde.

Descritores: Serviços de Saúde; Utilização de Instalações e Serviços; População Rural; População Urbana.
\end{abstract}

\begin{abstract}
Objective: to analyze the existence of urban-rural disparity in health services utilization by people living in a small municipality. Methods: cross-sectional study with a representative random sample of people aged 18 years and older in the urban area $(n=1.235)$ and the entire population of a rural district $(n=190)$. The Health Services Utilization questionnaire, from the National Household Survey in Brazil, was used. The analysis considered logistic regression models to examine factors associated with health services utilization within 30 days prior to the study. Results: the utilization rate by people in urban area was significantly higher than in rural area ( $45.0 \%$ vs $15.8 \%)$. Women and people who reported symptoms of diseases and diagnosis of hypertension were more likely to use health services. Conclusion: under the same municipal health organization, people living in the rural area have lower health services utilization when compared to those living in the urban area. Descriptors: Health Services; Facilities and Services Utilization; Rural Population; Urban Population.
\end{abstract}




\section{Introdução}

A utilização dos serviços de saúde há muito tempo vem sendo considerada um importante indicador da condição de saúde humana ${ }^{(1)}$. A esse respeito, apesar das condições de saúde da população brasileira ter melhorado consideravelmente nas últimas três décadas ${ }^{(2)}$, ainda persistem importantes diferenças entre pessoas que vivem em áreas rurais e pessoas que vivem em áreas urbanas ${ }^{(3-4)}$.

Embora muitos fatores, incluindo os socioeconômicos, contribuam para essas diferenças, o acesso aos serviços de saúde é um fator crucial ${ }^{(5)}$. Ainda hoje, passados 30 anos da atual Constituição Federal prever que a saúde é um direito de todos os cidadãos, além de um dever do Estado, garantido por meio de políticas sociais e econômicas direcionadas à redução dos riscos de doenças e agravos e ao acesso universal e igualitário aos cuidados e serviços, persistem sérias disparidades na utilização dos serviços de saúde ${ }^{(6)}$.

Já no início dos anos 1990, com a criação das Leis Orgânicas da Saúde, na tentativa de responder aos preceitos da nova Constituição, os municípios tornaram-se os responsáveis imediatos pela execução das principais ações e oferta de serviços para o acesso de seus cidadãos aos cuidados à saúde, configurando um modelo de gestão descentralizada. Mas, apesar de estudos de base domiciliar, e até de âmbito nacional, terem contribuído de forma inquestionável para a compreensão das disparidades urbano-rurais na utilização de serviços de saúde, as informações sobre a utilização dos serviços não têm sido consideradas pelos municípios no planejamento nem na alocação de recursos para a saúde ${ }^{(1)}$. Como um agravante, aqueles municípios de pequeno porte podem estar oferecendo condições igualmente restritivas ao acesso de pessoas vivendo tanto em áreas urbanas quanto em áreas rurais.

Diante dessa situação e considerando que a associação entre a utilização de serviços de saúde e a condição urbano-rural possa ficar mascarada nas avaliações em grande escala, entende-se ser necessá- rio explorar a questão sob a perspectiva de um mesmo sistema de saúde em município de pequeno porte. A avaliação da utilização de serviços que estão sob uma mesma organização municipal poderia, até certo ponto, balizar os indicadores e as características da oferta de serviços.

Entende-se que compreender a utilização dos serviços de saúde seja fundamental para o adequado planejamento da oferta de cuidados e para o monitoramento de uma cobertura universal e equânime dos serviços de saúde no âmbito dos municípios ${ }^{(7)}$. A compreensão das barreiras para a utilização de serviços no território municipal é essencial ao desenho de intervenções que visem ao aumento da cobertura assistencial. A eliminação das disparidades relacionadas ao acesso deve ser uma meta para melhorar o estado de saúde das populações e, para atingir essa meta, é necessário mensurar o problema no contexto local dos municípios. Assim, este estudo teve como objetivo analisar a existência de disparidade urbano-rural na utilização de serviços de saúde por pessoas vivendo em município de pequeno porte.

\section{Métodos}

Trata-se de um estudo epidemiológico transversal. Foram considerados indivíduos com 18 anos ou mais de idade, residentes em áreas urbana e rural de um município de pequeno porte localizado no Vale do Jequitinhonha, a aproximadamente $700 \mathrm{~km}$ da capital do estado de Minas Gerais, Brasil. 0 município, escolhido por ser campo de outras pesquisas do grupo que apresenta este trabalho, possuía uma população de pouco mais de 24 mil habitantes, sendo que 16.079 residiam na zona urbana. Aqueles que residiam na área rural, cerca de 30,0\%, estavam distribuídos em cinco distritos e comunidades adjacentes.

Sobre a estrutura de serviços de saúde no município, a área urbana possuía cinco unidades básicas de saúde e uma equipe da Estratégia Saúde da Família atuava em cada. $O$ atendimento à saúde da população rural era feito por uma equipe volante da Estratégia 
Saúde da Família, com atendimentos semanais ou quinzenais nas sedes das localidades. Agentes Comunitários de Saúde e Técnicos de Enfermagem permaneciam nas comunidades. 0 município possuía ainda um Centro de Atenção Psicossocial e, para a atenção hospitalar de baixa complexidade e atendimentos de urgência e emergência, dispunha de um hospital filantrópico, com regime de gestão privada. Sua referência macrorregional para a atenção de média e alta complexidade era Teófilo Otoni, cidade a $225 \mathrm{~km}$ de distância.

Na área urbana, a população estudada foi selecionada em três etapas a fim de se obter uma amostra representativa. Primeiramente, realizou-se um levantamento do número de domicílios cadastrados por unidades básicas de saúde, considerando dados do Sistema de Informação da Atenção Básica, seguido de cálculo da amostra mínima necessária representativa desses domicílios, que totalizou 1.229 domicílios (Tabela 1).

Tabela 1 - Cálculo amostral do número de domicílios a serem estudados na área urbana segundo Unidades Básicas de Saúde. Jequitinhonha, MG, Brasil, 2015

\begin{tabular}{lcc}
\hline $\begin{array}{l}\text { Unidades Bási- } \\
\text { cas de Saúde }\end{array}$ & $\begin{array}{c}\text { Número de domicílios } \\
\text { cadastrados }\end{array}$ & $\begin{array}{c}\text { Amostra mínima } \\
\text { de domicílios }\end{array}$ \\
\hline I & 1.450 & 304 \\
II & 560 & 228 \\
III & 702 & 248 \\
IV & 651 & 244 \\
V & 450 & 207 \\
Total & 3.813 & 1.229 \\
\hline
\end{tabular}

Para o cálculo amostral, realizado no programa Epi Info versão 6.0, considerou-se uma frequência esperada de $50 \%$, erro de $5 \%$ e intervalo de confiança de $95 \%$. Prevendo perdas, a amostra foi aumentada em 20,0\%, totalizando 1.475 domicílios urbanos; em seguida, realizou-se uma amostragem probabilística randomizada dos domicílios de cada área, considerando um nível de confiança de 95\%, no mesmo pro- grama Epi Info, a fim de selecionar aqueles a serem visitados. Na terceira etapa, por amostragem aleatória simples, foi sorteado, durante visita aos domicílios, um morador com idade igual ou maior que 18 anos em cada um dos domicílios selecionados, ainda que fosse preciso retornar à residência para a realização da entrevista, considerando até três tentativas, caso o sorteado não se encontrasse presente. As perdas somaram 240 domicílios/indivíduos e foram motivadas por recusas e por não encontrar o indivíduo selecionado após as três tentativas. Ao final, a amostra urbana contou com 1.235 participantes, sendo um para cada domicílio visitado.

$\mathrm{Na}$ área rural, todos os indivíduos na faixa etária estudada que residiam em um dos distritos, distante $70 \mathrm{~km}$ da área urbana, foram convidados para participar. A população elegível era de 218 moradores, distribuídos em 176 domicílios. 0 critério para a seleção da amostra rural diferiu do urbano devido às características semelhantes da oferta de serviços à população rural, com apenas uma Equipe de Saúde percorrendo todas as localidades, segundo um mesmo modelo de atenção. As perdas na área rural totalizaram 28 pessoas, sendo os motivos recusa e não ter sido encontrado após três tentativas. Assim, a população rural estudada foi composta por 190 pessoas.

Os dados foram coletados no período de janeiro a dezembro de 2010. Utilizou-se questionário para obter informações demográficas (sexo e idade), socioeconômicas (escolaridade, renda familiar mensal, número de pessoas na família, recebimento de auxílio financeiro governamental e posse de plano de saúde) e condições de saúde (autopercepção de problemas com a saúde ou sintomas de doenças nos 30 dias anteriores à entrevista, autopercepção do estado de saúde geral e diagnóstico autorreferido de doenças crônicas). Para os dados sobre a utilização de serviços de saúde nos 30 dias anteriores à entrevista, utilizou-se o questionário do suplemento sobre Acesso e Utilização de Serviços de Saúde no Brasil da Pesquisa Nacional por Amostra de Domicílios. A equipe de co- 
leta de dados foi composta por alunos de graduação e pós-graduação da Escola de Enfermagem da Universidade Federal de Minas Gerais, previamente treinados, sendo utilizados equipamentos portáteis e digitais de coleta (Personal Digital Assistant - modelo Dell-Axim X50) que permitiram a transferência imediata dos dados coletados para um banco de conferência, ainda na área de estudo. Com isso, realizaram-se avaliações preliminares dos dados e identificação de erros e ausência de informações, possibilitando o retorno imediato aos domicílios para correção ou verificação das incoerências quando necessário. A transferência dos dados dos equipamentos de coleta para os softwares de análise foi realizada em duplicidade com posterior comparação visando eliminar a possibilidade de erros no processo.

A variável dependente (desfecho) na análise dos dados foi a utilização de serviços de saúde nos 30 dias anteriores à entrevista, sem distinção se eram serviços públicos ou privados. Os participantes foram classificados em duas categorias: aqueles que utilizaram algum serviço de saúde e aqueles que não utilizaram serviços de saúde no período.

As variáveis independentes foram selecionadas com base em um modelo comportamental de utilização de serviços de saúde defendido desde a década de 1970 e ainda frequentemente utilizado ${ }^{(8)}$. Como variáveis relacionadas aos fatores predisponentes, adotaram-se idade, sexo e educação; as relacionadas aos fatores facilitadores, renda familiar, auxílio financeiro governamental, cobertura por plano de saúde e local de moradia urbano-rural; e aos fatores relacionados com o estado de saúde, adotaram-se a percepção do estado geral de saúde, a presença de sintomas de doenças nos últimos 30 dias e autorrelato de diagnósticos de doenças crônicas (hipertensão arterial sistêmica ou outras).

As análises foram feitas utilizando o software estatístico Statistical Package for the Social Sciences for Windows, versão 17.0. Inicialmente, para comparar a utilização de serviços de saúde, bem como as variáveis explicativas entre os residentes nas áreas urbana e rural, utilizou-se o teste chi-quadrado. Posteriormente, modelos de regressão logística foram utilizados para estudar as possíveis disparidades urbano-rurais na utilização de serviços de saúde, controlando-os pelas variáveis de confusão $0^{(8)}$. 0 nível de significância estatística foi estabelecido em $5 \%(\mathrm{p}<0,05)$.

O estudo foi aprovado pelo Comitê de Ética em Pesquisa da Universidade Federal de Minas Gerais sob o Parecer no 0174.0.203.000-10.

\section{Resultados}

Participou do estudo um total de 1.425 pessoas (1.235 na área urbana e 190 na rural). A taxa de utilização dos serviços de saúde pelas pessoas residentes na área urbana foi significativamente maior que a taxa de utilização dos residentes da área rural $(45,0 \%$ vs $15,8 \%, p<0,001$ ) (Tabela 2). Em comparação aos participantes rurais, os da área urbana foram representados por um número maior de mulheres, de pessoas mais velhas e com maior escolaridade, maior renda familiar mensal per capta e maior cobertura por planos de saúde. Encontrou-se maior proporção de pessoas da área urbana com diagnóstico de hipertensão arterial sistêmica $(36,7 \%$ vs $23,2 \%, \mathrm{p}<0,001)$. A autopercepção de sintomas que indicassem problemas de saúde nos 30 dias antes da entrevista, bem como a autorreferência a estado de saúde geral regular, ruim ou muito ruim no mesmo período ou a outras doenças crônicas, não foi diferente entre as pessoas das duas áreas.

Dois diferentes modelos de regressão logística multivariada foram considerados para a análise da utilização de serviços de saúde (Tabela 3). 0 modelo básico (modelo 1) considerou somente a variável localização (urbano/rural). No modelo completo (modelo 2), todas as variáveis independentes foram inseridas na análise de regressão com a variável localização. 
Tabela 2 - Estatística descritiva da população estudada segundo o local de residência (urbana e rural).

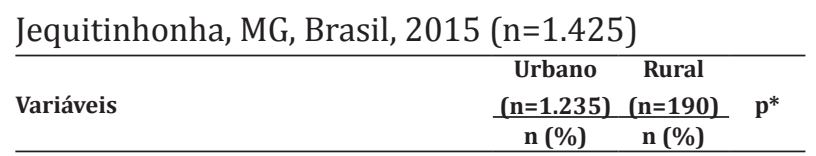

Variável dependente

Utilização de serviços de saúde nos últimos

30 dias

$556(45,0) 30(15,8)<0,001$

Variáveis independentes

Sexo, feminino

Idade, $\geq 50$ anos

$791(64,0) 99(52,1) \quad 0,002$

Escolaridade, 0 a 4 anos de estudos $575(46,6) \quad 71(37,4) \quad 0,018$

Renda familiar per capta, $\leq 1 / 2$ Salário Mí-

nimo

Família recebe auxílio financeiro governa-

mental

Possui plano de saúde

Autopercepção de problemas de saúde nos

últimos 30 dias

Autopercepção do estado de saúde como $397(32,1) 72(37,9) \quad 0,116$ regular, ruim ou muito ruim

Hipertensão arterial sistêmica autorreferida $453(36,7) 44(23,2)<0,001$

Outra doença crônica ${ }^{\dagger}$

$170(13,8) \quad 19(10,0) \quad 0,154$

*Teste chi-quadrado foi usado para comparar a utilização de serviços de saúde, bem como as variáveis independentes entre os residentes em área urbana e rural; ${ }^{\dagger}$ exceto hipertensão arterial sistêmica

Tabela 3 - Análise de regressão logística para a utilização de serviços de saúde. Jequitinhonha, MG, Brasil, $2015(\mathrm{n}=1.425)$

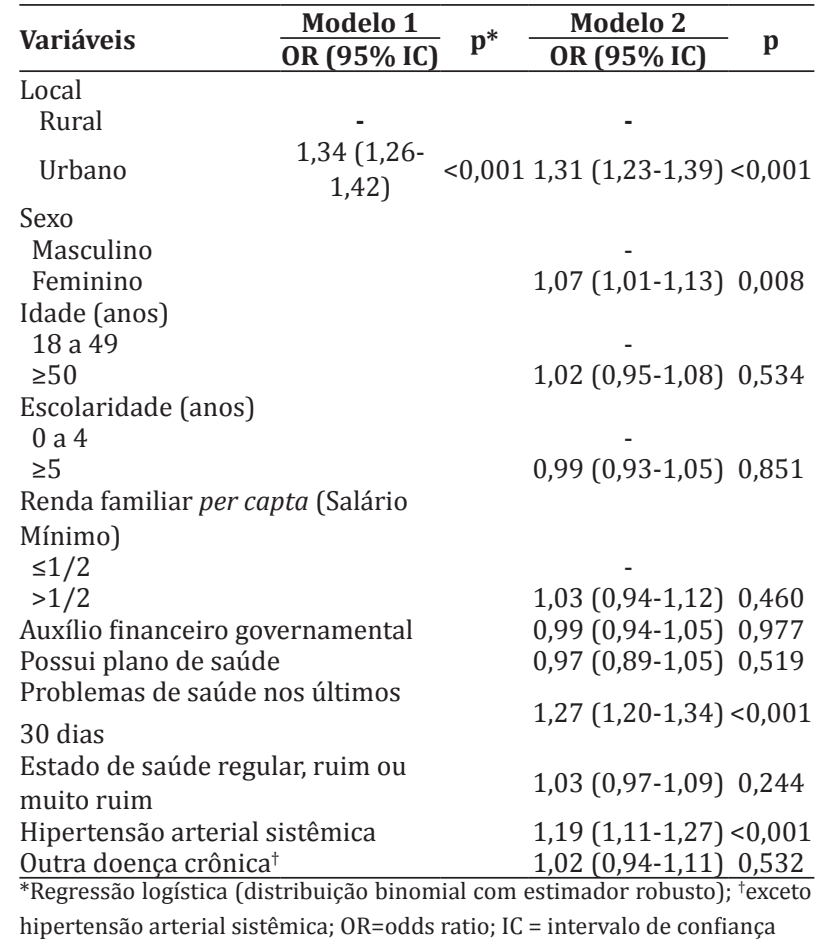

No modelo 1 de regressão logística, que considerou somente o local de residência, evidenciou-se que os moradores da área urbana tiveram mais chance de utilizarem os serviços de saúde $(\mathrm{OR}=1,34$; IC 95\%: 1,26-1,42). Após ajustar para todas as variáveis independentes estudadas, no modelo 2, o odds ratio para residência em área urbana diminuiu de 1,34 para 1,31, mas mantendo a significância estatística $(\mathrm{p}<0,001)$. Isso indica que os residentes na área urbana tiveram mais chances de utilizarem os serviços de saúde (OR=1,31; IC 95\%: 1,23-1,39), mesmo após controlar a análise por possíveis variáveis de confusão. Adicionalmente, as mulheres, pessoas que perceberam os sintomas de doenças e as que afirmaram possuir o diagnóstico de hipertensão arterial sistêmica também utilizaram mais os serviços de saúde.

\section{Discussão}

Embora o acesso aos serviços de saúde no Brasil tenha aumentado ao longo das três últimas décadas ${ }^{(9)}$, este estudo confirmou que ainda existem disparidades na utilização. Pessoas que vivem em área rural utilizam menos os serviços de saúde do que as de área urbana de um mesmo município. Antes de avançar na discussão dos resultados, cabe informar que uma das limitações assumidas pelo estudo é que se compara a utilização entre as duas áreas com uma variável dicotômica urbano-rural, desconsiderando os variados graus de ruralidade. Estudos que usam um indicador binário para rural-urbano são menos propensos a observarem diferenças nas medidas de acesso que aqueles consideram um contínuo urbano-rural gradual e mais específico. Também, este estudo utiliza a morbidade autorreferida para mensurar doenças crônicas, não adotando nenhum meio para verificação ou conferência da informação, incorrendo no viés de informação. Contudo, há evidências da validade dos resultados que utilizam medidas de indivíduos que se declaram portadores de doenças crônicas ${ }^{(10)}$. Outras características não incluídas nesta pesquisa podem também ter tido influência na diferença de utilização 
identificada, tais como as relacionadas aos serviços, e devem ser consideradas em futuros estudos.

A magnitude das diferenças na utilização de serviços entre populações urbanas e rurais depende, entre outros fatores, da medida de acesso utilizada e de como se caracterizam os ambientes rural e urbano. Mas, em geral, a maioria dos estudos realizados, seja no Brasil ou em outros países, encontrou essa mesma relação de iniquidade ${ }^{(7-8)}$.

A fundamentação para se considerar o contexto do sistema municipal de saúde para a análise de iniquidades no acesso aos serviços de saúde reside no fato de que as estratégias de oferta são desenhadas pelos gestores municipais sem um modelo normativo claro de estratégia. Nem mesmo a Política Nacional de Atenção Básica estabelece as diretrizes para a organização da Atenção Básica destinada às pessoas vivendo em áreas rurais ${ }^{(11)}$. Nesse contexto descentralizado de atenção, a informação sobre o acesso e a utilização de serviços pela população rural é crítica para uma gestão local da saúde eficiente, particularmente em municípios com precários indicadores de saúde ${ }^{(1)}$.

Atualmente, de um modo geral, os municípios brasileiros adotam estratégias distintas para garantir o acesso dos cidadãos rurais e urbanos à saúde. Enquanto os serviços de atenção básica urbanos costumam contar com médicos, enfermeiros e outros profissionais diariamente durante a semana, nas áreas rurais os atendimentos são esporádicos - semanais, quinzenais, mensais ou mesmo não periódicos. Em muitas áreas, os atendimentos, quando ocorrem, são focados exclusivamente nas consultas médicas. Situações nas quais técnicos em enfermagem atuam sem a supervisão de enfermeiros não são raras nas áreas rurais, podendo colocar em risco a saúde das pessoas ${ }^{(12)}$. A compreensão dos determinantes e fatores associados à utilização é fundamental na concepção e oferta de serviços cuidadosamente direcionados, podendo determinar economia dos escassos recursos financeiros municipais, além de possibilitar melhoria na saúde da população ${ }^{(1)}$. Entre os desafios mais recentes dos governos municipais e que justificarão ainda mais o cuidado com a utilização dos serviços, destacam-se as transformações das condições de saúde da população e os efeitos do congelamento dos gastos sociais por 20 anos desde 2016 no Brasil ${ }^{(13)}$.

Além da inadequada infraestrutura e menor disponibilidade de serviços para cuidados à saúde, as grandes distâncias a serem percorridas, as dificuldades de transporte e a baixa renda são fatores que, associados, podem contribuir para a redução na utilização de serviços de saúde pelas pessoas vivendo em áreas rurais ${ }^{(5)}$. Barreiras impostas pela "inacessibilidade" geográfica e indisponibilidade de serviços nas áreas rurais já foram descritas como os mais importantes preditores da utilização por pessoas rurais ${ }^{(5)}$.

Dos fatores estudados relacionados à predisposição, sexo foi o único significativamente associado com a utilização. Como outros estudos ${ }^{(8)}$, este mostrou que as mulheres utilizam mais os serviços de saúde do que os homens. Existem várias possíveis explicações para essa constatação ${ }^{(14)}$. Dentre estas, as diferenças na utilização referentes ao sexo podem estar relacionadas às atitudes individuais de homens e mulheres, muitas vezes estereotipadas, perante as próprias vulnerabilidades e a percepção de bem-estar, além das necessidades relacionadas à reprodução. Também, os serviços públicos de saúde, dos quais depende a maioria absoluta das pessoas estudadas, promovem maior oferta de atendimentos preventivos para as mulheres do que para os homens. Entretanto, é necessário destacar que a população urbana estudada apresentou significativamente mais mulheres que a rural, podendo ter se comportado como uma variável de confusão na análise.

A utilização também esteve relacionada com o estado de saúde das pessoas, reconhecidamente o fator mais importante na determinação da procura por atendimento à saúde na área urbana ou no meio rural $^{(9)}$. De modo geral, pessoas com hipertensão, em virtude da necessidade de controle, colaboraram para elevar a demanda e utilização dos serviços de saúde. Particularmente, os serviços públicos de saúde no Brasil destinam considerável oferta de cuidados à po- 
pulação com hipertensão, acarretando maior utilização por essas pessoas ${ }^{(15)}$. A maior prevalência de pessoas com hipertensão autorreferida na área urbana pode ter contribuído para a utilização mais frequente por essa população. Esse achado, que indica menor prevalência de hipertensão nas pessoas da área rural, corrobora com outros estudos ${ }^{(16-17)}$ e pode estar relacionado à maior dificuldade para acesso ao diagnóstico, e não, necessariamente, com melhor condição de saúde. A maior dificuldade de acesso aos serviços de saúde nos momentos de necessidade percebida pode favorecer o uso de plantas medicinais e práticas de cuidado à saúde culturalmente fundamentadas em crenças e religiosidade no campo, interferindo no curso da doença ${ }^{(18)}$.

Mesmo diante dos avanços acumulados após a instituição do Sistema Único de Saúde ${ }^{(9)}$, é necessário admitir que o acesso e a utilização dos serviços de saúde por pessoas nas áreas rurais do Brasil ainda estão muito aquém do necessário em uma sociedade com um sistema universal de saúde. Como foi demonstrado, a população rural é privada das capacidades e oportunidades para cuidar da saúde que desejaria ter, prerrogativas fundamentais para se alcançar o verdadeiro desenvolvimento humano. Cabe mencionar que, muito provavelmente, a situação de desigualdades retratada é um problema organizacional e estrutural dos municípios e que vem de longa data. Essa diferença entre as áreas urbanas e rurais sugere que políticas de acesso devem ser fortalecidas com o objetivo de anular as iniquidades em saúde.

\section{Conclusão}

Apesar da Constituição Federal prever o acesso universal e igualitário aos serviços de atenção à saúde, o presente estudo mostrou existir disparidade urbano-rural na utilização desses serviços por pessoas vivendo em um mesmo município de pequeno porte. Pessoas residentes em área rural utilizam menos serviços de saúde quando comparadas àquelas da área urbana, mesmo quando estão sob a mesma organização municipal de saúde.

\section{Colaborações}

Vieira EWR, Dutra IR e Cerqueira LJ contribuíram na análise e interpretação dos dados, redação do artigo e aprovação final da versão a ser publicada. Gazzinelli A contribuiu na concepção e projeto, revisão crítica relevante do conteúdo intelectual e aprovação final da versão a ser publicada.

\section{Referências}

1. Ngugi AK, Agoi F, Mahoney MR, Lakhani A, Mang'Ong'o D, Nderitu E, et al. Utilization of health services in a resourcelimited rural area in Kenya: prevalence and associated household-level factors. PLoS One. 2017; 12(2): e0172728. doi: https://doi.org/10.1371/journal.pone.0172728

2. Souza MFM, Malta DC, Franca EB, Barreto ML. Changes in health and disease in Brazil and its States in the 30 years since the Unified Healthcare System (SUS) was created. Ciênc Saúde Coletiva. 2018; 23(6):1737-50. doi: http://dx.doi. org/10.1590/1413-81232018236.04822018

3. Nepomuceno GA, Matoso LF, Miranda WJB, Lima TF, Gazzinelli A, Vieira EW. Anthropometric indicators associated with high blood pressure in children living in urban and rural areas. Rev Latino-Am Enfermagem. 2019; 27:e3150. doi: http:// dx.doi.org/10.1590/1518-8345.2760-3150

4. Confortin SC, Antes DL, Pessini J, Jayce I, Schneider C, Orsi E, et al. Comparação do perfil socioeconômico e condições de saúde de idosos residentes em áreas predominantemente rural e urbana da Grande Florianópolis, Sul do Brasil. Cad Saúde Coletiva. 2016; 24(3):330-8. doi: http:// dx.doi.org/10.1590/1414-462x201600030034

5. Oladipo JA. Utilization of health care services in rural and urban areas: A determinant factor in planning and managing health care delivery systems. Afr Heal Sci. 2014; 14(2):322-33. doi: http://dx. doi.org/10.4314/ahs.v14i2.6

6. Fernandes NFS, Galvão JR, Assis MMA, Almeida $\mathrm{PF}$, Santos AM. Acesso ao exame citopatológico do colo do útero em região de saúde: mulheres invisíveis e corpos vulneráveis. Cad Saúde Pública. 2019; 35(10):e00234618. doi: http://dx.doi. org/10.1590/0102-311x00234618 
7. Dorjdagva J, Batbaatar E, Svensson M, Dorjsuren B, Batmunkh B, Kauhanen J. Free and universal, but unequal utilization of primary health care in the rural and urban areas of Mongolia. Int J Equity Health. 2017; 16(1):73. doi: http://dx.doi. org/10.1186/s12939-017-0572-4

8. Liu X, Li N, Liu C, Ren X, Liu D, Gao B, et al. Urbanrural disparity in utilization of preventive care services in China. Medicine (Baltimore). 2016; 95(37):e4783. doi: http://dx.doi.org/10.1097/ MD.0000000000004783

9. Viacava F, Oliveira RAD, Carvalho CC, Laguardi J, Bllido JG. SUS: supply, access to and use of health services over the last 30 years. Ciênc Saúde Coletiva. 2018; 23(6):1751-62. doi: http://dx.doi. org/10.1590/1413-81232018236.06022018

10. Lima-Costa MF, Peixoto SV, Firmo JOA. Validity of self-reported hypertension and its determinants (the Bambuí study). Rev Saúde Pública. 2004; 38(5):637-42. doi: http://dx.doi.org/10.1590/ S0034-89102004000500004

11. Ministério da Saúde (BR). Portaria $n^{\circ} 2.436$, de 21 de Setembro de 2017. Aprova a Política Nacional de Atenção Básica, estabelecendo a revisão de diretrizes para a organização da Atenção Básica, no âmbito do Sistema Único de Saúde (SUS) [Internet]. 2017 [citado 2019 out 23]. Disponível em: http://bit.ly/3655Fw3

12. Conselho Federal de Enfermagem (BR). Em zona rural, Coren-MT garante atendimento de enfermagem seguro [Internet]. 2018 [citado 2019 out 23]. Disponível em: http://bit.ly/3044kmu

13. Rasella D, Basu S, Hone T, Paes-Sousa R, Ocké-Reis CO, Millett C. Child morbidity and mortality associ- ated with alternative policy responses to the economic crisis in Brazil: a nationwide microsimulation study. PLoS Med. 2018; 15(5):e1002570. doi: https://doi.org/10.1371/journal.pmed.1002570

14. Levorato CD, Mello LM, Silva AS, Nunes AA. Fatores associados à procura por serviços de saúde numa perspectiva relacional de gênero. Ciênc Saúde Coletiva. 2014; 19(4):1263-74. doi: http://dx.doi. org/10.1590/1413-81232014194.01242013

15. Barreto MS, Mendonça RD, Pimenta AM, Garcia-Vivar C, Marcon SS. Non-use of primary care routine consultations for individuals with hypertension. Ciênc Saúde Coletiva. 2018; 23(3):795804.doi:http://dx.doi.org/10.1590/141381232018233.12132016

16. Katchunga $P B$, Mirindi $P$, Baleke A, Twagirumukiza M. The trend in blood pressure and hypertension prevalence in the general population of South Kivu between 2012 and 2016 results from two representative cross-sectional surveys - the Bukavu observational study. PLoS One. 2019; 14(8):e0219377. doi: http://dx.doi.org/10.1371/ journal.pone.0219377

17. Lamelas P, Diaz R, Orlandini A, Avezum A, Oliveira G. Prevalence, awareness, treatment and control of hypertension in rural and urban communities in Latin American countries. J Hypertens. 2019; 37(9):1813-21. doi: http://dx.doi.org/10.1097/ HJH.0000000000002108

18. Rückert B, Cunha DM, Modena CM. Healthcare knowledge and practices of the rural population: an integrative literature review. Interface (Botucatu). 2018; 22(66):903-14. doi: http://dx.doi. org/10.1590/1807-57622017.0449 